\title{
Escalas para evaluar la calidad de vida en personas con enfermedad renal crónica avanzada: Revisión integrativa
}

\author{
Ana Julia Carrillo-Algara ${ }^{1}$, Germán Antonio Torres-Rodríguez², Cesar Steven Leal-Moreno², Sandra Milena \\ Hernández-Zambrano ${ }^{3}$
}

${ }^{1}$ Enfermera Magister Administración en Salud. Profesora Titular. Facultad de Enfermería. Grupo Perspectivas del Cuidado. Fundación Universitaria de Ciencias de la Salud. Bogotá. Colombia

${ }^{2}$ Enfermero, Especialista en Enfermería nefrológica del adulto. Fundación Universitaria de Ciencias de la Salud. Bogotá, Colombia

${ }^{3}$ Enfermera. PhD en Ciencias de la Salud. Mg en cuidados de salud para la promoción de la autonomía de las personas y la atención a los procesos de fin de vida. Docente Investigadora. Facultad de Enfermería. Grupo Perspectivas del Cuidado. Fundación Universitaria de Ciencias de la Salud. Bogotá. Colombia

\section{Resumen}

Introducción: La calidad de vida es un indicador de bienestar percibido por personas diagnosticadas con enfermedad renal crónica avanzada, al respecto la literatura reporta diferentes instrumentos para su medición y de algunos de ellos describe las características psicométricas y adaptación transcultural.

Objetivo: Identificar los instrumentos reportados por la literatura para evaluar la calidad de vida en la persona con enfermedad renal crónica avanzada y describir las características psicométricas y validación transcultural de los mismos.

Material y Método: Se realizó una revisión integrativa de la literatura científica publicada en el periodo 20132018 , en español, inglés y portugués. La búsqueda se realizó en 2 etapas, utilizando los operadores booleanos AND y OR, y los términos DeCS y MeSH para identificar escalas que evalúan la calidad de vida de los pacientes con enfermedad renal crónica avanzada, posteriormente se realizó una búsqueda focalizada para identificar cuales contaban con adaptación transcultural y las características psicométricas de las mismas.

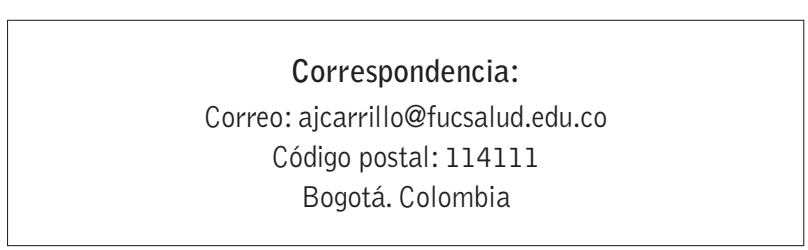

Resultados: La búsqueda inicial reportó 364 títulos elegibles, se seleccionaron 20, que comprendían instrumentos genéricos y específicos, de ellos 5 escalas se han usado en pacientes con enfermedad renal crónica avanzada: SF-12, SF-36, KDQOL 36, WHOQOL BREF, WHOQOL OLD.

Conclusión: La escala KDQOL 36, posee dimensiones específicas para evaluar al paciente con enfermedad renal crónica avanzada, se encuentra adaptada transculturalmente en varios países de habla hispana y sus características psicométricas son confiables, el Alfa de Cronbach es $>0,7$, y su utilidad es adecuada porque es de fácil aplicación, poco compleja y a un bajo costo.

PALABRAS CLAVE: calidad de vida; enfermedades renales; diálisis renal; trasplante de riñón; tratamiento conservador.

\section{Scales for assessing the quality of life in people with advanced chronic kidney disease: Integrative review}

\section{Abstract}

Introduction: Quality of life is an indicator of well-being perceived by people diagnosed with advanced chronic kidney disease. In this respect, literature reports about different instruments for measurement and some 
of them describes the psychometric characteristics and cross-cultural validation.

Objective: To identify the instruments reported by the literature to evaluate the quality of life in persons with advanced chronic kidney disease and to describe theeir psychometric characteristics and cross-cultural validation.

Material and Method: An integrative review of the scientific literature in Spanish, English and Portuguese, published between 2013-2018, was carried out. The search was conducted in two stages using the Boolean operators AND and OR, and DeCS and MeSH terms to identify scales assessing quality of life of patients with advanced chronic kidney disease, subsequently a targeted search was performed to identify which had cross-cultural validation and their psychometric characteristics.

Results: The initial search reported 364 eligible titles, 20 were selected, which included generic and specific instruments, of which 5 scales were used in patients with advanced chronic kidney disease: SF-12, SF-36, KDQOL 36, WHOQOL BREF, WHOQOL OLD.

Conclusion: The KDQOL 36 scale has specific dimensions to evaluate the patient with advanced chronic kidney disease, is cross-culturally adapted in several Spanish-speaking countries and its psychometric characteristics are reliable, Cronbach alpha is $>0.7$, and its usefulness is suitable because it is easy to apply, unsophisticated and at a low cost.

KEYWORDS: quality of life; kidney diseases; renal dialysis; renal transplant; conservative treatment.

\section{Introducción}

La Enfermedad Renal Crónica Avanzada (ERCA) es un problema creciente en Colombia y el mundo, cuya morbilidad y mortalidad van en ascenso $0^{1}$. Es una patología no transmisible que incluye los estadios 3 y 4 de la ERC, cursando con descenso grave del filtrado glomerular ( $F G<30 \mathrm{ml} / \mathrm{min})$, por tanto, los objetivos terapéuticos están orientados a la reducción de complicaciones, y preparación de Terapias de Reemplazo Renal (TRR)². Esta situación crónica, avanzada y progresiva, afecta la calidad de vida de la persona y su familia, el número de años de vida perdidos, los hábitos y aumenta costos para el paciente y el sistema de salud, por tanto se considera una enfermedad de alto costo ${ }^{1}$. Las personas diagnosticadas con ERCA que se encuen- tran en Terapias de Reemplazo Renal (TRR) pueden tener complicaciones generadas por el tratamiento 0 por la enfermedad propiamente dicha, que aumentan el número de hospitalizaciones, generan síntomas desagradables y deterioran la calidad de vida ${ }^{3}$.

En 1975 se describió calidad de vida como un proceso dinámico y cambiante de bienestar que difiere según el sistema de valores de las personas, y en 1994 el grupo World Health Organization Quality of Life (WHOQOL) constituido por la Organización Mundial de la Salud (OMS) la conceptualiza como "la percepción individual de la propia posición en la vida dentro del contexto del sistema cultural y de valores en que se vive y en relación con sus objetivos, esperanzas, normas y preocupacio$n e s^{1 / 3}$. Desde entonces es considerada como un indicador de calidad y eje fundamental para la toma de decisiones ${ }^{3}$, está íntimamente relacionada con la morbimortalidad, y es entendida como la evaluación que hace la persona respecto a su salud y nivel de funcionamiento para realizar actividades de la vida diaria, incluye, funciones físicas, psicológicas, sociales, percepción general de la salud, movilidad y bienestar emocional entre otras ${ }^{4}$.

En pacientes con ERCA en TRR o terapia conservadora, se han utilizado instrumentos para evaluar la calidad de vida, que incluyen las dimensiones físicas, psicológicas y sociales que no pueden observarse o medirse de una forma directa, y se basan en la percepción (subjetiva) del sujeto ${ }^{5}$, obtenida de la forma más objetiva y rigurosa posible, minimizando errores de medición que afecten la validez de los datos, y generen sesgos de información que distorsionen la correlación entre el resultado medido y la realidad del fenómeno ${ }^{6}$. Por tanto, el objetivo del estudio es identificar los instrumentos para evaluar la calidad de vida en el paciente con ERCA, que reporta la literatura y describir las características psicométricas y validación transcultural de los mismos.

\section{Material y Método}

Se realizó una revisión integrativa que busca sintetizar el conocimiento con un enfoque sistemático y riguros $0^{7}$. La revisión se realizó por etapas: en la primera se identificaron las escalas más utilizadas para medir calidad de vida en pacientes con ERCA, para ello se establecieron 3 ecuaciones de búsqueda utilizando palabras clave según los descriptores MeSH y DeCS (Quality of Life, Kidney Diseases, SCALE, Dialysis, kidney transplantation, Conservative Treatment) en las diferentes combi- 
naciones posibles con los operadores booleanos (AND, $O R$ ) en las bases de datos: CINAHL, CUIDEN, MEDLINE, NURSING OVID, y metabuscador BIBLIOTECA VIRTUAL DE SALUD. Como criterios de inclusión se tomaron artículos publicados del 2013 al 2018, en inglés, portugués, o español (Figura 1). En la segunda etapa se realizó búsqueda focalizada con el fin de identificar las características psicométricas y la validación transcultural de las escalas seleccionadas. Se buscó en las mismas bases de datos de la etapa 1, en google académico y en el repositorio de tesis doctorales TESEO; con las ecuaciones: SF12 AND Validación, SF36 AND Validación, KDOQOL 36 AND Validación, WHOQOL BREF AND Validación WHOQOL OLD AND Validación. Se determinó como criterio de inclusión los instrumentos que contarán con versión en español y adaptación transcultural en países iberoamericanos.

En la primera etapa una vez efectuada la búsqueda se realizó filtro por título, y selección por resumen, como resultado se identificaron estudios que utilizaron las mismas herramientas para evaluar la calidad de vida de pacientes con ERCA, los cuales fueron el insumo para la segunda etapa, en la cual se realizó lectura crítica de los estudios de validación y adaptación transcultural con una lista de chequeo que contiene los criterios de la guía de Validación de escalas de medición en salud propuesta por Sánchez y Echeverry ${ }^{8}$, y evaluación independiente para valorar la calidad metodológica recogiendo los criterios de validez, confiabilidad y utilidad.

Para caracterizar la revisión y hacer un análisis de los instrumentos se elaboraron 3 tablas; en la Tabla 1, se muestra los artículos identificados, población a la cual se aplicó y el instrumento que se utilizó; en la Tabla 2, se describe información detallada de los puntos clave de cada instrumento, que parámetros que evalúa, en cuanto tiempo se administra, si es guiada o auto dirigida y países en los cuales ha sido adaptada transculturalmente, en la Tabla 3 se presentan las propiedades psicométricas en relación a la reproducibilidad, validez, sensibilidad, y utilidad, características principales de las escalas en salud definidos como ${ }^{8}$ :

- Reproducibilidad se evalúa a través de la fiabilidad, es el grado en que una escala es capaz de medir sin errores, permite establecer las verdaderas diferencias entre la población a estudiar.

- Validez es la capacidad que tiene el instrumento de medir para lo cual fue diseñado, esta caracte-

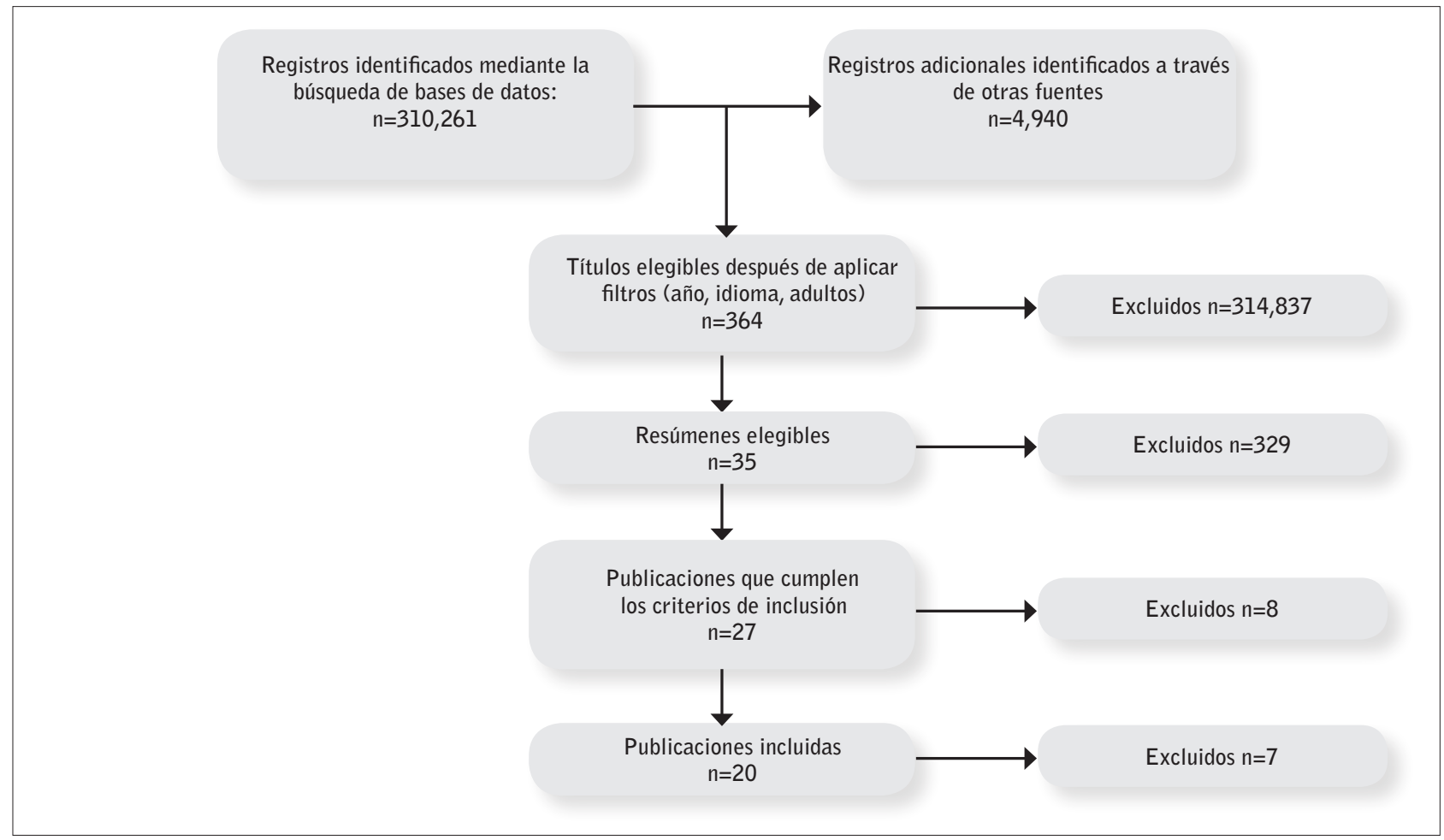

Figura 1. Diagrama de identificación y selección de artículos.

Fuente: The PRISMA statement. PLoS medicine ${ }^{6}$, adaptada por los autores. 
rística posee unas propiedades como componente y son: validez de apariencia, que hace referencia al grado en que las preguntas aplicadas evalúan de forma lógica lo que se pretende medir. La validez de contenido pretende evaluar si las preguntas incluidas pertenecen al dominio correspondiente.

- Sensibilidad: capacidad que posee el instrumento para detectar cambios a través del tiempo.

- Utilidad: su aplicación resulta fácil, y económica.

Desde el punto de vista ético el presente estudio se considera sin riesgo según lo estipulado en la resolución 8430 de 1993 por ser una revisión de literatura y se rige por la ley 44 de 1993 donde se esclarece la responsabilidad del investigador con relación de la protección al derecho de autor de los artículos utilizados en este documento mediante la adecuada referenciación bibliográfica.

\section{Resultados}

La búsqueda inicial reportó 364 títulos elegibles, tras la lectura de resúmenes y aplicabilidad de criterios de inclusión y exclusión se seleccionaron 20 , que permitieron identificar instrumentos de medición genéricos y específicos entre ellos 5 escalas usadas en pacientes con ERCA: SF-12, SF-36, KDQOL 36, WHOQOL BREF, WHOQOL OLD (Tabla 1).

En salud existen múltiples escalas para evaluar la calidad de vida ${ }^{9}$ concepto subjetivo que comprende actitudes, creencias, adaptación, ajuste social entre otros $^{10,11}$. Gran parte de ellas se han desarrollado en países de habla inglesa y su uso en lenguajes y culturas diferentes implica hacer la traducción, el ajuste lingüístico y el proceso de validación ${ }^{9,11}$.

Tabla 1. Caracterización de la muestra bibliográfica.

\begin{tabular}{|c|c|c|c|c|c|c|}
\hline $\begin{array}{l}\text { REFERENCIA } \\
\text { BIBLIOGRÁFICA }\end{array}$ & $\begin{array}{l}\text { IDIOMA DEL } \\
\text { ARTÍ́CULO }\end{array}$ & $\begin{array}{l}\text { PAÍS EN QUE SE } \\
\text { REALIZÓ EL ESTUDIO }\end{array}$ & OBJETIVO PRINCIPAL & $\begin{array}{l}\text { DISEÑO } \\
\text { METODOLÓGICO }\end{array}$ & POBLACIÓN & INSTRUMENTO \\
\hline $\begin{array}{l}\text { Simpson clusters } \\
\text { in patients on } \\
\text { dialysis and their } \\
\text { association with } \\
\text { quality-of-life } \\
\text { outcomes }^{12} \text {. }\end{array}$ & Inglés. & California. & $\begin{array}{l}\text { Explorar e identificar los } \\
\text { grupos de síntomas (síntomas } \\
\text { coexistentes) en pacientes } \\
\text { en diálisis y sus posibles } \\
\text { asociaciones con los síntomas } \\
\text { depresivos y los resultados de } \\
\text { la calidad de vida relacionada } \\
\text { con la salud (CVRS). }\end{array}$ & $\begin{array}{l}\text { Descriptivo } \\
\text { transversal. }\end{array}$ & $\begin{array}{l}301 \text { pacientes } \\
\text { en diálisis. }\end{array}$ & KDQOL, SF-36. \\
\hline $\begin{array}{l}\text { Traducción, } \\
\text { validación y } \\
\text { confiabilidad:la } \\
\text { forma corta } \\
\text { (KDQOL-SF Form) } \\
\text { para medir Calidad } \\
\text { de vida en de las } \\
\text { enfermedades del } \\
\text { riñón Herramienta } \\
\text { en urdu }{ }^{13} \text {. }\end{array}$ & Inglés. & Pakistán. & $\begin{array}{l}\text { Traducir, validar y evaluar } \\
\text { la confiabilidad la forma } \\
\text { abreviada (KDQOL-SF-36) } \\
\text { en urdu, idioma nacional de } \\
\text { Pakistán. Para medir calidad } \\
\text { de vida en la enfermedad } \\
\text { renal. }\end{array}$ & $\begin{array}{l}\text { Estudio descriptivo } \\
\text { transversal } \\
\text { multicultural. }\end{array}$ & $\begin{array}{l}130 \\
\text { Pacientes con } \\
\text { enfermedad } \\
\text { renal en etapa } \\
\text { terminal, y } \\
\text { pacientes con } \\
\text { insuficiencia } \\
\text { renal aguda. }\end{array}$ & KDQ0L, SF-36. \\
\hline $\begin{array}{l}\text { Relación entre las } \\
\text { complicaciones y } \\
\text { la calidad de vida } \\
\text { del paciente en } \\
\text { hemodiálisis } 3 \text {. }\end{array}$ & Español. & México. & $\begin{array}{l}\text { Evaluar la calidad de vida de } \\
\text { pacientes en hemodiálisis y } \\
\text { determinar la asociación entre } \\
\text { las complicaciones y la CV. }\end{array}$ & $\begin{array}{l}\text { Estudio transversal } \\
\text { analítico. }\end{array}$ & $\begin{array}{l}157 \text { pacientes } \\
\text { en HD mayores } \\
\text { de } 18 \text { años y } \\
\text { con más de } \\
3 \text { meses en } \\
\text { tratamiento. }\end{array}$ & KDQ0L-36. \\
\hline $\begin{array}{l}\text { Cultural relevance } \\
\text { of the quality- } \\
\text { of-life tools for } \\
\text { people with kidney } \\
\text { failure }{ }^{14} \text {. }\end{array}$ & Inglés. & Barcelona. & $\begin{array}{l}\text { Examinar la relevancia } \\
\text { cultural de dos herramientas } \\
\text { (SF-36 y el índice de QOL). }\end{array}$ & Estudio descriptivo. & $\begin{array}{l}161 \text { pacientes } \\
\text { en diálisis } \\
\text { y } 350 \\
\text { personas de la } \\
\text { comunidad. }\end{array}$ & SF 36, Índice Q0L. \\
\hline
\end{tabular}




\begin{tabular}{|c|c|c|c|c|c|c|}
\hline $\begin{array}{l}\text { REFERENCIA } \\
\text { BIBLIOGRÁFICA }\end{array}$ & $\begin{array}{l}\text { IDIOMA DEL } \\
\text { ARTÍCULO }\end{array}$ & $\begin{array}{l}\text { PAÍS EN QUE SE } \\
\text { REALIZÓ EL ESTUDIO }\end{array}$ & OBJETIVO PRINCIPAL & $\begin{array}{l}\text { DISEÑO } \\
\text { METODOLÓGICO }\end{array}$ & POBLACIÓN & INSTRUMENTO \\
\hline $\begin{array}{l}\text { Estudio } \\
\text { comparativo } \\
\text { del estado } \\
\text { físico, mental y } \\
\text { percepción de } \\
\text { calidad de vida } \\
\text { relacionada con } \\
\text { la salud de los } \\
\text { pacientes en } \\
\text { diálisis }^{15} \text {. }\end{array}$ & Español. & España. & $\begin{array}{l}\text { Valorar y analizar la } \\
\text { percepción de salud, la } \\
\text { capacidad funcional, el estado } \\
\text { nutricional y psicológico de } \\
\text { los pacientes de hemodiálisis } \\
\text { (HD) y diálisis peritoneal } \\
\text { (DP). }\end{array}$ & Estudio descriptivo. & $\begin{array}{l}42 \text { pacientes } \\
\text { ( } 21 \text { en } \\
\text { hemodiálisis y } \\
21 \text { en diálisis } \\
\text { peritoneal). }\end{array}$ & Cuestionario SF-12. \\
\hline $\begin{array}{l}\text { Psychometric } \\
\text { Properties of the } \\
\text { Kidney Disease } \\
\text { Quality of Life-36 } \\
\text { Questionnaire } \\
(\text { KDQ0L-36 }\end{array}$ & Inglés. & Hong Kong. & $\begin{array}{l}\text { Evaluar la validez, } \\
\text { confiabilidad y sensibilidad de } \\
\text { los ítems específicos de calidad } \\
\text { de vida-36 y enfermedad } \\
\text { renal (KDQOL-36) en } \\
\text { pacientes chinos sometidos a } \\
\text { diálisis de mantenimiento. }\end{array}$ & Estudio descriptivo. & $\begin{array}{l}\text { Un total de } \\
110 \text { pacientes } \\
\text { en diálisis y } \\
122 \text { pacientes } \\
\text { con trasplante } \\
\text { renal fueron } \\
\text { reclutados. }\end{array}$ & KDQ0L-36. \\
\hline $\begin{array}{l}\text { Validación de } \\
\text { los componentes } \\
\text { específicos de la } \\
\text { enfermedad de la } \\
\text { enfermedad renal } \\
\text { Calidad de vida-36 } \\
\text { (KDQOL-36) } \\
\text { en pacientes } \\
\text { chinos sometidos } \\
\text { a diálisis de } \\
\text { mantenimiento }{ }^{17} \text {. }\end{array}$ & Inglés. & China. & $\begin{array}{l}\text { Determinar la validez y } \\
\text { la fiabilidad de la versión } \\
\text { en chino cantonés del } \\
\text { cuestionario Quality of } \\
\text { Life-36 (KDQOL-36 TM) de } \\
\text { la enfermedad renal. La } \\
\text { escala se ha traducido al } \\
\text { chino cantonés, pero no se ha } \\
\text { probado entre las poblaciones } \\
\text { de habla cantonesa. }\end{array}$ & $\begin{array}{l}\text { Estudio de } \\
\text { correlaciòn. }\end{array}$ & $\begin{array}{l}356 \text { pacientes } \\
\text { chinos } \\
\text { sometidos } \\
\text { a diálisis de } \\
\text { mantenimiento. }\end{array}$ & KDQ0L-36. \\
\hline $\begin{array}{l}\text { ¿Es la escala, } \\
\text { calidad de vida } \\
\text { de la enfermedad } \\
\text { renal-36 } \\
\text { (KDQ0L-36) un } \\
\text { instrumento válido } \\
\text { para pacientes en } \\
\text { diálisis chinos? }{ }^{18} \text {. }\end{array}$ & Inglés. & Hong kong. & $\begin{array}{l}\text { Determinar la validez y } \\
\text { la fiabilidad de la versión } \\
\text { en chino cantonés del } \\
\text { cuestionario Quality of Life- } 36 \\
\text { (KDQOL-36 TM) chino entre } \\
\text { la población de Hong Kong de } \\
\text { habla cantonesa y } \\
\text { Pacientes chinos de Kong } \\
\text { sometidos a diálisis. Los } \\
\text { resultados } \\
\text { puede contribuir a valiosas } \\
\text { aplicaciones clínicas y } \\
\text { proporcionar } \\
\text { una comparación internacional } \\
\text { de la calidad de vida } \\
\text { entre la población de habla } \\
\text { cantonesa. } \\
\text { La escala se ha traducido al } \\
\text { chino cantonés, pero no se ha } \\
\text { probado entre las poblaciones } \\
\text { de habla cantonesa. }\end{array}$ & $\begin{array}{l}\text { Investigación } \\
\text { cuantitativa, } \\
\text { transversal y } \\
\text { descriptiva. }\end{array}$ & $\begin{array}{l}110 \text { pacientes } \\
\text { en diálisis y } \\
122 \text { pacientes } \\
\text { con trasplante } \\
\text { renal fueron } \\
\text { reclutados. }\end{array}$ & $\begin{array}{l}\text { KDQ0L-36. Version } \\
\text { corta. }\end{array}$ \\
\hline $\begin{array}{l}\text { Quality of life of } \\
\text { elderly people } \\
\text { with chronic } \\
\text { kidney disease } \\
\text { in conservative } \\
\text { treatment. Revista } \\
\text { Brasileira De } \\
\text { Enfermagem }^{19}\end{array}$ & Inglés. & Brasil. & $\begin{array}{l}\text { Describir la calidad de } \\
\text { vida (CV) de ancianos } \\
\text { con Enfermedad Renal } \\
\text { Crónica (ERC) en } \\
\text { tratamiento conservador, } \\
\text { correlacionando con aspectos } \\
\text { sociodemográficos y de salud. }\end{array}$ & $\begin{array}{l}\text { Investigación } \\
\text { cuantitativa, } \\
\text { transversal y } \\
\text { descriptiva. }\end{array}$ & $\begin{array}{l}\text { Participaron } 35 \\
\text { ancianos }(54,30 \% \\
\text { mujeres) con edad } \\
\text { media de } 68,26 \\
\text { años. En promedio } \\
\text { se midieron } 3,70 \\
\text { comorbilidades y } \\
5,60 \text { complicacio- } \\
\text { nes relacionadas } \\
\text { con ERC. }\end{array}$ & $\begin{array}{l}\text { WHOQOL-Bref y } \\
\text { WHOQOL-Old. }\end{array}$ \\
\hline
\end{tabular}




\begin{tabular}{|c|c|c|c|c|c|c|}
\hline $\begin{array}{l}\text { REFERENCIA } \\
\text { BIBLIOGRÁFICA }\end{array}$ & $\begin{array}{l}\text { IDIOMA DEL } \\
\text { ARTÍCULO }\end{array}$ & $\begin{array}{l}\text { PAÍS EN QUE SE } \\
\text { REALIZÓ EL ESTUDIO }\end{array}$ & OBJETIVO PRINCIPAL & $\begin{array}{l}\text { DISEÑO } \\
\text { METODOLÓGICO }\end{array}$ & POBLACIÓN & INSTRUMENTO \\
\hline $\begin{array}{l}\text { Transcultural } \\
\text { adaptation and } \\
\text { validation of the } \\
\text { Mexican version of } \\
\text { the kidney disease } \\
\text { questionnaire } \\
\text { KDQOL-SF36 } \\
\text { version } 1.3^{20} \text {. }\end{array}$ & Inglés. & México. & $\begin{array}{l}\text { Traducir el cuestionario } \\
\text { de calidad de vida de la } \\
\text { Enfermedad Renal } 36 \\
\text { (KDQOL-SF36) v1.3 al } \\
\text { idioma español, adaptarlo } \\
\text { culturalmente y validarlo en la } \\
\text { población mexicana. }\end{array}$ & Estudio descriptivo. & $\begin{array}{l}194 \\
\text { mexicanos en } \\
\text { hemodiálisis. }\end{array}$ & KDQ0L, SF-36. \\
\hline $\begin{array}{l}\text { Measuring health- } \\
\text { related quality } \\
\text { of life in patients } \\
\text { with conservatively } \\
\text { managed stage } \\
5 \text { chronic kidney } \\
\text { disease }^{21} \text {. }\end{array}$ & Inglés. & Londres e Inglaterra. & $\begin{array}{l}\text { Determinar la capacidad } \\
\text { de respuesta al cambio de } \\
\text { SF-36 en pacientes con ERC } \\
\text { en estadio } 5 \text { administrada de } \\
\text { forma conservadora. }\end{array}$ & Estudio descriptivo. & $\begin{array}{l}74 \text { persona } \\
\text { formaron la } \\
\text { muestra final } \\
\text { para el estudio. }\end{array}$ & SF-36. \\
\hline $\begin{array}{l}\text { Psychometric } \\
\text { Properties of } \\
\text { the Patient's } \\
\text { Perception of Life } \\
\text { on Hemodialysis } \\
\text { Scale }\end{array}$ & Inglés. & Canadá. & $\begin{array}{l}\text { Evaluar la fiabilidad y validez } \\
\text { de la Escala de Percepción de } \\
\text { Hemodiálisis del Paciente. }\end{array}$ & $\begin{array}{l}\text { Diseño de corte } \\
\text { transversal. }\end{array}$ & $\begin{array}{l}\text { Pacientes } \\
5236 .\end{array}$ & SF-36. \\
\hline $\begin{array}{l}\text { Quality of life } \\
\text { related to the } \\
\text { health of chronic } \\
\text { renal failure } \\
\text { patients on } \\
\text { dialysis }^{23} \text {. }\end{array}$ & Inglés. & Brasil. & $\begin{array}{l}\text { Evaluar la calidad de vida } \\
\text { relacionada con la salud de } \\
\text { pacientes con insuficiencia } \\
\text { renal crónica en diálisis. }\end{array}$ & Estudio transversal. & $\begin{array}{l}101 \text { pacientes } \\
\text { con ERC que } \\
\text { habían estado } \\
\text { en tratamiento } \\
\text { de diálisis } \\
\text { durante tres } \\
\text { meses. }\end{array}$ & SF-12. \\
\hline $\begin{array}{l}\text { Validation and } \\
\text { reliability of the } \\
\text { South Indian } \\
\text { (Kannada) version } \\
\text { of the Kidney } \\
\text { Disease and } \\
\text { Quality of Life } \\
(\text { KDQOL-36) })^{24} \text {. }\end{array}$ & Inglés. & India. & $\begin{array}{l}\text { Evaluar la validez y fiabilidad } \\
\text { del sur de la India (Canarés) } \\
\text { Versión del instrumento } \\
\text { KDQOL-36 para pacientes } \\
\text { en HD. }\end{array}$ & Estudio transversal. & $\begin{array}{l}82 \text { pacientes de } \\
\text { mantenimiento } \\
\text { de alta } \\
\text { definición. }\end{array}$ & KDQ0L-36. \\
\hline $\begin{array}{l}\text { Validation of the } \\
\text { Kidney Disease } \\
\text { Quality of Life } \\
\text { Short Form } 36 \\
\text { (KDQOL-36TM }^{25} \text {. }\end{array}$ & Inglés. & Estados Unidos. & $\begin{array}{l}\text { Evaluar la confiabilidad y } \\
\text { validez de la Forma Corta } \\
36 \text { de Calidad de Vida } \\
\text { de la Enfermedad Renal } \\
\text { (KDQOL-36 TM) en hispanos } \\
\text { con ERC de leve a moderada. }\end{array}$ & Estudio transversal. & 420 hispanos. & KDQ0L-36. \\
\hline $\begin{array}{l}\text { Análisis de la } \\
\text { calidad de vida } \\
\text { del paciente en la } \\
\text { etapa prediálisis }{ }^{26} \text {. }\end{array}$ & Español. & México. & $\begin{array}{l}\text { Analizar la Calidad de Vida } \\
\text { Relacionada con la Salud } \\
\text { de los pacientes con ERC } \\
\text { Avanzada en estadio } 5 .\end{array}$ & $\begin{array}{l}\text { Observacional } \\
\text { descriptivo } \\
\text { transversal. }\end{array}$ & $\begin{array}{l}\text { Se estudiaron } \\
32 \text { pacientes. }\end{array}$ & KDQ0L-SF.36. \\
\hline $\begin{array}{l}\text { Determining } \\
\text { the validity and } \\
\text { reliability of the } \\
\text { Chinese version } \\
\text { of the Kidney } \\
\text { Disease Quality of } \\
\text { Life Questionnaire } \\
(\text { KDQOL-36 }\end{array}$ & Español. & China. & $\begin{array}{l}\text { Evaluar la validez y fiabilidad } \\
\text { de la KDQOL-36 TM con una } \\
\text { muestra de pacientes chinos. }\end{array}$ & Estudio descriptivo. & $\begin{array}{l}103 \text { pacientes } \\
\text { con ERC. }\end{array}$ & KDQ0L-36. \\
\hline
\end{tabular}




\begin{tabular}{|c|c|c|c|c|c|c|}
\hline $\begin{array}{l}\text { REFERENCIA } \\
\text { BIBLIOGRÁFICA }\end{array}$ & $\begin{array}{l}\text { IDIOMA DEL } \\
\text { ARTÍCULO }\end{array}$ & $\begin{array}{l}\text { PAÍS EN QUE SE } \\
\text { REALIZÓ EL ESTUDIO }\end{array}$ & OBJETIVO PRINCIPAL & $\begin{array}{l}\text { DISEÑO } \\
\text { METODOLÓGICO }\end{array}$ & POBLACIÓN & INSTRUMENTO \\
\hline $\begin{array}{l}\text { Short-Form } 120 \\
\text { Short-Form } 36 \\
\text { para medir los } \\
\text { cambios en la } \\
\text { calidad de vida } \\
\text { en pacientes en } \\
\text { diálisis ?28. }\end{array}$ & Inglés. & Estados Unidos. & $\begin{array}{l}\text { Evaluar la capacidad de } \\
\text { SF-12 versus SF-36 para } \\
\text { detectar cambios a lo largo } \\
\text { del tiempo y la asociación de } \\
\text { SF-12 versus SF-36 con la } \\
\text { mortalidad a corto y largo } \\
\text { plazo en pacientes en diálisis. }\end{array}$ & $\begin{array}{l}\text { Estudio de } \\
\text { seguimiento } \\
\text { prospectivo entre } \\
\text { pacientes con } \\
\text { diálisis incidente. }\end{array}$ & $\begin{array}{l}1379 \text { pacientes } \\
\text { con diálisis } \\
\text { incidente. }\end{array}$ & $\begin{array}{l}\text { SF-12 versus } \\
\text { SF-36. }\end{array}$ \\
\hline $\begin{array}{l}\text { Adaptación } \\
\text { transcultural del } \\
\text { cuestionario kdqol } \\
\text { SF } 36 \text { para evaluar } \\
\text { calidad de vida en } \\
\text { pacientes con ERC } \\
\text { en Colombia } 29\end{array}$ & Español. & Colombia. & $\begin{array}{l}\text { Adaptar transculturalmente, } \\
\text { para el uso en Colombia, del } \\
\text { cuestionario para determinar } \\
\text { calidad de vida en pacientes } \\
\text { con ERCterminal, KDQOL- } \\
\text { SF-36 Kidney Disease Quality } \\
\text { of Life (instrumento mixto). }\end{array}$ & $\begin{array}{l}\text { Descriptivo } \\
\text { transversal. }\end{array}$ & $\begin{array}{l}\text { Grupo } \\
\text { conformado por } \\
12 \text { mujeres y } \\
18 \text { hombres, la } \\
\text { edad tuvo una } \\
\text { media de } 48,7 \\
\text { años. }\end{array}$ & KDQOL, SF-36. \\
\hline
\end{tabular}

Fuente: Elaboración propia.

Tabla 2. Caracterización de instrumentos (dimensiones, aplicabilidad, administración).

\begin{tabular}{|c|c|c|c|}
\hline INSTRUMENTO & DIMENSIONES O AREAS & ADMINISTRACIÓN & $\begin{array}{l}\text { ADAPTACIÓN TRANSCULTURAL } \\
\text { Y UTILIZACIÓN POR PAÍSES }\end{array}$ \\
\hline SF-12 & $\begin{array}{l}8 \text { dimensiones: } \\
\text { - Función Física. } \\
\text { - Función Social. } \\
\text { - Rol físico. } \\
\text { - Rol Emocional. } \\
\text { - Salud mental. } \\
\text { - Vitalidad. } \\
\text { - Dolor corporal. } \\
\text { - Salud General. } \\
12 \text { ítems en total. }\end{array}$ & $\begin{array}{l}\text { Cuestionario auto administrado, } \\
\text { también se puede administrar } \\
\text { por entrevistador de forma } \\
\text { personal, telefónica o mediante } \\
\text { soporte informático. } \\
\text { El tiempo de cumplimentación } \\
\text { es de }<2 \text { minutos }{ }^{31} \text {. } \\
\text { Es aplicable a población general } \\
\text { sana o enferma. }\end{array}$ & $\begin{array}{l}\text { - España. } \\
\text { Escala adaptada y traducida al español, pero la } \\
\text { literatura solamente reporta que se encuentra validada } \\
\text { en España. }\end{array}$ \\
\hline SF-36 & $\begin{array}{l}8 \text { dimensiones: } \\
\text { - Funcionamiento Físico. } \\
\text { - Limitación por problemas } \\
\text { físicos. } \\
\text { - Dolor corporal. } \\
\text { - Funcionamiento o rol social. } \\
\text { - Salud mental. } \\
\text { - Limitación por problemas } \\
\text { emocionales. } \\
\text { - Vitalidad, energía o fatiga. } \\
\text { - Percepción general de la salud. } \\
12 \text { ítems en total. }\end{array}$ & $\begin{array}{l}\text { El cuestionario debe ser auto } \\
\text { administrado. } \\
\text { Su duración es variable de } 5 \text { a } \\
10 \text { minutos. } \\
\text { Herramienta genérica aplicable } \\
\text { a población general }>15 \text { años }{ }^{32} \text {. }\end{array}$ & $\begin{array}{l}\text { - España. } \\
\text { - Colombia. } \\
\text { - México. } \\
\text { - Chile. } \\
\text { - Perú. } \\
\text { - Ecuador. } \\
\text { - Argentina. } \\
\text { - Brasil. }\end{array}$ \\
\hline WHOQOL BREF & $\begin{array}{l}4 \text { dimensiones: } \\
\text { - Salud física. } \\
\text { - Salud psicológica. } \\
\text { - Relaciones sociales. } \\
\text { - Ambiente. } \\
26 \text { ítems en total. }\end{array}$ & $\begin{array}{l}\text { El cuestionario debe ser auto } \\
\text { administrado. } \\
\text { Cuando la persona no sea capaz } \\
\text { de leer o escribir por razones de } \\
\text { educación,cultura o salud puede } \\
\text { ser entrevistada. } \\
\text { Duración }<5 \text { minutos } \\
\text { El tiempo de referencia } \\
\text { que contempla el WHOQ0L } \\
\text { es de dos semanas, } \\
\text { retrospectivamente. } \\
\text { Herramienta genérica, aplicable } \\
\text { a cualquier población }>15 a^{2} 0^{33} \text {. }\end{array}$ & $\begin{array}{l}\text { - Chile. } \\
\text { Durante la búsqueda no se evidencian más países con } \\
\text { adaptación transcultural para esta herramienta. }\end{array}$ \\
\hline
\end{tabular}




\begin{tabular}{|c|c|c|c|}
\hline INSTRUMENTO & DIMENSIONES O AREAS & ADMINISTRACIÓN & $\begin{array}{l}\text { ADAPTACIÓN TRANSCULTURAL } \\
\text { Y UTILIZACIÓN POR PAIISES }\end{array}$ \\
\hline WHOQOL OLD & $\begin{array}{l}6 \text { dimensiones: } \\
\text { - Capacidad sensorial. } \\
\text { - Autonomía. } \\
\text { - Actividades pasadas presentes } \\
\text { y futuras. } \\
\text { - Participación/aislamiento. } \\
\text { - Intimidad. } \\
\text { - Muerte y agonía. } \\
24 \text { ítems en total. }\end{array}$ & $\begin{array}{l}\text { El cuestionario debe ser } \\
\text { preferiblemente auto } \\
\text { administrado. } \\
\text { No existen limitantes si el } \\
\text { cuestionario de realiza a modo } \\
\text { de entrevista. } \\
\text { Duración }<5 \text { minutos. } \\
\text { Herramienta específica para } \\
\text { adultos mayores de } 65 \text { años }^{30} \text {. }\end{array}$ & $\begin{array}{l}\text { - México. } \\
\text { - Chile. } \\
\text { - España. }\end{array}$ \\
\hline KDQOL 36 & $\begin{array}{l}11 \text { dimensiones específicas: } \\
\text { - Síntomas/problemas. } \\
\text { - Efectos de la enfermedad renal } \\
\text { en la vida diaria. } \\
\text { - Carga de la enfermedad. } \\
\text { - Situación laboral. } \\
\text { - Función cognitiva. } \\
\text { - Relaciones sociales. } \\
\text { - Función sexual. } \\
\text { - Sueño. } \\
\text { - Apoyo social. } \\
\text { - Actitud del personal de diálisis. } \\
\text { - Satisfacción del paciente. } \\
43 \text { ítems en total. }\end{array}$ & $\begin{array}{l}\text { El cuestionario debe ser auto } \\
\text { administrado. } \\
\text { Ella duración del cuestionario } \\
\text { es variable y está por encima de } \\
\text { los } 10 \text { minutos. } \\
\text { Herramienta específica para } \\
\text { población con enfermedad } \\
\text { renal }{ }^{29} \text {. }\end{array}$ & $\begin{array}{l}\text { - México. } \\
\text { - Colombia. } \\
\text { - Brasil. } \\
\text { - Chile. } \\
\text { - España. }\end{array}$ \\
\hline
\end{tabular}

Fuente: Elaboración propia.

Tabla 3. Características psicométricas de los instrumentos en relación a la reproducibilidad, validez, sensibilidad, y utilidad.

\begin{tabular}{|c|c|c|c|c|c|c|c|c|}
\hline CRITERIO & DEFINICIÓN & ESTADÍSTICO & $\begin{array}{l}\text { RESULTADO } \\
\text { SATISFACTORIO }\end{array}$ & SF-12 & SF-36 & $\begin{array}{l}\text { WHOQOL } \\
\text { BREF }\end{array}$ & $\begin{array}{l}\text { WHOQOL } \\
\text { OLD }\end{array}$ & $\begin{array}{l}\text { KDOQOL } \\
36\end{array}$ \\
\hline $\begin{array}{l}\text { Reproducibi- } \\
\text { lidad }\end{array}$ & $\begin{array}{l}\text { Produce, } \\
\text { consistentemente los } \\
\text { mismos resultados, } \\
\text { cuando se aplica a } \\
\text { los mismos sujetos en } \\
\text { diferentes periodos de } \\
\text { tiempo }{ }^{34} \text {. }\end{array}$ & $\begin{array}{l}\text { Coeficiente alfa de } \\
\text { Cronbach }^{34} \text {. }\end{array}$ & $\geq 0,7^{34}$ & $\begin{array}{l}0,63 \\
\text { físico } \\
0,72 \\
\text { Mental }{ }^{31} \text {. }\end{array}$ & $\begin{array}{l}0,58 \\
\text { mental } \\
0,99 \\
\text { físico } \\
\end{array}$ & $0,88^{33}$ & $0,80^{30}$ & $\begin{array}{l}0,80 \\
0,87^{29}\end{array}$ \\
\hline Validez & $\begin{array}{l}\text { Mide lo que realmente } \\
\text { queremos medir }{ }^{34} \text {. }\end{array}$ & $\begin{array}{l}\text { Correlación } \\
\text { de Pearson o } \\
\text { de Spearman, } \\
\text { Coeficiente alfa de } \\
\text { Cronbach }^{34} \text {. }\end{array}$ & $\begin{array}{l}\text { Entre } 0,4 \text { y } 0,70 \\
>0,77^{34}\end{array}$ & $>0,7$ & $\begin{array}{l}0,71 \\
0,94\end{array}$ & - & - & - \\
\hline Sensibilidad & $\begin{array}{l}\text { Capacidad de un } \\
\text { instrumento para } \\
\text { detectar cambios a } \\
\text { través del tiempo }{ }^{34} \text {. }\end{array}$ & $\begin{array}{l}\text { Coeficiente alfa de } \\
\text { Cronbach }^{34} \text {. }\end{array}$ & - & $>0,7$ & - & - & - & - \\
\hline Utilidad & $\begin{array}{l}\text { La es escala es de } \\
\text { fácil aplicación, poca } \\
\text { compleja y bajo } \\
\text { costo } \\
\text { 33. }\end{array}$ & $\begin{array}{l}\text { No describe } \\
\text { estadístico }{ }^{33} \text {. }\end{array}$ & N/A & $\begin{array}{l}\text { Sujeto al } \\
\text { estudio a } \\
\text { realizar. }\end{array}$ & - & - & - & - \\
\hline
\end{tabular}


Los instrumentos para evaluar la calidad de vida en paciente con ERCA se pueden clasificar como genéricos y específicos dependiendo a la población a la cual este dirigido, los genéricos son instrumentos que se pueden utilizar en diferentes pacientes y su diseño pretende obtener un aspecto de calidad de vida que pueda aplicarse en distintos contextos, como es el caso de la escala SF-36, SF-12, WHOQOL BREF; los específicos pueden serlo para una enfermedad en concreto, o por grupo etario con diferentes alteraciones ${ }^{8}$, éstas últimas son las características de las escalas KDQOL 36 y WHOQOL OLD ${ }^{29,30}$. En los 19 estudios encontrados se evidenció que KDQOL 36 se aplicó en 11 investigaciones de corte transversal, con muestras poblacionales desde 30 pacientes hasta 356. La escala SF36 fue utilizada en 7 estudios con muestras entre 32 hasta 5536 pacientes en estudios transversales; la escala SF-12 se usó en 4 estudios transversales con muestras entre 42 y 1279 pacientes y solo en un estudio descriptivo se utilizó WHOQOL BREF y WHOQOL OLD en 15 pacientes. En algunos estudios se utilizaron 2 herramientas.

Los instrumentos seleccionados varían en número de ítems, tiempo y aplicabilidad a diferentes grupos poblacionales (Tabla 2), no obstante están enfocados a categorizar el bienestar biopsicosocial del individuo; una de las herramientas específicas es idónea para evaluar la población con ERC.

En la Tabla 2 se especifican los tiempos empleados para administrar el cuestionario y la forma como debe aplicarse (auto administrado o dirigido), además se identifican los países donde se ha utilizado, sin embargo escalas como la SF-12, WHOQOL BREF, WHOQOL OLD, son cuestionarios que se han aplicado en países de habla hispana pero no se ha realizado el proceso de adaptación transcultural a diferencia de la SF-36 y la KDQ0L 36 que son de amplio uso y han sido adaptadas transculturalmente en diferentes países de habla hispana.

En la Tabla 3 se describen las características psicométricas de los instrumentos que se identificaron en los estudios de validación.

Con relación a los instrumentos genéricos, la escala SF-36 muestra unas características psicométricas de reproducibilidad de 0,99 el rol físico, función física y rol emocional obtuvieron mejores resultados de fiabilidad en comparación con el apartado de función social donde fue de 0,58. Es importante señalar que esta herramienta no evalúa trastornos de sueño, función cognitiva, la función familiar o la función sexual. $Y$ se han descrito problemas en su aplicación a personas adultas mayores por que no contestan o dejan espacios en blanco por lo tanto es importante la supervisión en el momento de administrar el cuestionario para evitar problemas de interpretación y diligenciamiento: El SF-12 hasta el momento no ha determinado su validez para la utilización en la práctica clínica, por lo tanto se desconoce si un buen instrumento para detectar cambios en la calidad de vida a través del tiempo, pero ha sido utilizado como herramienta genérica y en poblaciones específicas como pacientes con ERCA y muestra una alta consistencia interna y fiabilidad $^{35}$. EI instrumento WHOQOL-BREF reportó una reproducibilidad de 0,88 determinada mediante Alfa de Cronbach, está disponible para su aplicabilidad en diferentes culturas y, poblaciones en todo el proceso salud enfermedad. Lo cual es fundamental a la hora de considerar un instrumento transculturalmente y poder comparar resultados entre países. En cuanto a los instrumentos específicos, la escala WHOQoL-OLD ha demostrado propiedades psicométricas adecuadas y consistentes en su versión en español, con Alfa de Cronbach 0,80; en población brasileña el Alfa de Cronbach es de 0,71 a 0,88 y en población chilena es de 0,83. Es utilizada específicamente en adultos mayores debido a que posee un módulo donde se relaciona la experiencia del envejecimiento. EI instrumento KDQOL-36 posee consistencia interna y fiabilidad determinada por Alfa de Cronbach entre 0,80 y 0,87 por tanto es una herramienta válida y fiable para evaluar la calidad de vida en pacientes con ERCA, en su componente de efectos de enfermedad renal reporta 0,83 con la correlación más fuerte en la subescala de síntomas / problemas.

\section{Discusión}

Las escalas que miden Calidad de Vida Relacionada con la Salud (CVRS) son habitualmente cuestionarios que incluyen los componentes físicos, emocionales, cognitivos, sociales, las percepciones de salud/bienestar y las perspectivas futuras y se clasifican en genéricos que evalúan la CVRS en la población en general o grupo etario, y específicos que se focalizan en una enfermedad o problema de salud ${ }^{34}$.

La literatura científica reporta numerosos instrumentos utilizados en población con ERCA (SF-12, SF-36, KDOQOL 36, WHOQOL BREF, WHOQOL OLD), com- 
puestos por una serie de ítems o preguntas agrupadas en dimensiones que miden diferentes aspectos de la salud. No se reportan herramientas que cumplan con todas las características psicométricas, o no se describe de forma detallada su sensibilidad y utilidad, además unas han tenido más adaptaciones transculturales que otras.

Las herramientas que miden la calidad de vida relacionada con la salud y son adaptadas transculturalmente deben cumplir con los criterios de traducción, retrotraducción, revisión de expertos y prueba piloto para garantizar una adecuada aceptación y validez en su ámbito de aplicación ${ }^{35}$. En algunas ocasiones, se considera que la traducción y adaptación de un instrumento de utilización habitual en determinado campo, garantiza la conservación de sus propiedades psicométricas; sin embargo, es imperativa su adaptación sociocultural y validación ${ }^{35}$.

Otras características que debe tener una escala de medición en salud son la sencillez, viabilidad y aceptación por parte del paciente e investigadores además de poseer las propiedades psicométricas de fiabilidad $\mathrm{y}$ validez las cuales son determinantes en todo instrumento de medición ${ }^{35}$.

La OMS diseñó una herramienta para evaluar calidad de vida de forma genérica. El World Health Organization Quality of Life versión breve (WHOQOL-BREF), con buenas características psicométricas y adaptación transcultural en distintos idiomas, que se ha aplicado en numerosos estudios a nivel mundial tanto a personas sanas como enfermas, por tanto se puede afirmar que es una herramienta válida para evaluar la perspectiva del paciente, obtener información relevante, y desarrollar estudios experimentales y obervacionales $^{35}$.

La "evaluación internacional de la calidad de vida" (International Quality of Life Assessment Project, IQOLA) fue creada en 1991, con la finalidad de traducir, adaptar y aprobar la aplicabilidad transcultural del instrumento (SF-36), cuestionario que evalúa la calidad de vida relacionada con salud y ha sido validado para la población en general y poblaciones específicas incluyendo pacientes con ERCA abordando su componente físico y mental.

La mayor parte de los instrumentos que miden calidad de vida son aplicables a la población general resultando útiles en sujetos sanos o enfermos, es decir son gené- ricas, la KDQOL es una escala mixta, permite evaluar componentes para población en general pero además tiene un nivel específico para ERC sin importar el tipo de tratamiento que esté recibiendo el paciente, es uno de los que más tiene publicaciones de validación y adaptación en distintos idiomas a nivel mundial ${ }^{29}$. Otro instrumento específico es la WHOQoL-Old que posee un módulo para adulto mayor que evalúa aspectos específicos del envejecimiento ${ }^{30}$. SF-12 y SF-36 son instrumentos diseñados para población general y pacientes con una edad mínima de 14 años ${ }^{31-32}$, KDQ0L 36 es una forma abreviada de la SF-12 de forma general y la carga de la enfermedad renal, síntomas, y problemas de la enfermedad, no se evidencia una edad mínima para su aplicabilidad ${ }^{29}$.

Algunas herramientas son la versión abreviada de otras, fueron creadas y avaladas con el fin de disminuir los tiempos de respuesta y ocasionar así mayor aceptación y facilidad en la aplicación. Este es el caso de la herramienta SF-12, pero la más utilizada en investigación y evaluación de resultados clínicos ha sido el formato de 36 ítems. Siendo esta una escala con características psicométricas más confiables, con un Alfa de Cronbach > a 0,7 y mayor índice de adaptación transcultural a nivel mundial ${ }^{31}$.

Las diferentes escalas que miden la calidad de vida postuladas en este estudio tienen grados de confiabilidad aceptables, la mayoría reportan un Alfa de Cronbach $>0,7$, exceptuando la escala SF-12 que presenta datos con ligera desviación a la izquierda $(<0,7)$; la viabilidad en la utilización de cada escala se ve diferenciada en la utilidad (en que se enfoca la herramienta) y en la validación transcultural que posea la escala en el país donde se realizará el estudio ${ }^{31}$.Las escalas que poseen mejores propiedades psicométricas en cuanto a reproducibilidad y validez son las herramientas SF-36 y KDQ0L 36, cuya alfa de cronbach es $>0,8$ y se han realizado procesos rigurosos de adaptación transcultural en múltiples países de habla hispana; sin embargo la única herramienta que posee dimensiones específicas para personas con enfermedad renal es la KDQOL 36, aportando así un grado de utilidad mayor al evaluar calidad de vida en pacientes con ERCA ${ }^{31}$, además reporta mejores características psicométricas.

El proceso de validación de un instrumento se afianza son su uso y la realización de estudios en diferentes ámbitos y poblaciones que garanticen documentar su validez ${ }^{32}$. Dentro de las limitaciones de esta revisión 
se encontró la falta de información clara frente la sensibilidad y utilidad de las distintas escalas, además pocos estudios reportan las características psicométricas de forma detallada.

Como líneas futuras de investigación se sugiere la realización de estudios que evalúen de forma rigurosa todas las características psicométricas de las escalas, dado que en lo encontrado no se reporta de forma completa datos de sensibilidad y utilidad. Es importante que los instrumentos para evaluar la calidad de vida estén validados y adaptados transculturalmente en el país donde se va a implementar, esto permite una medición de las variables de forma rigurosa y objetiva, ya que los errores en la medición pueden afectar la validez de los datos, y generar sesgos de información en el momento de la recolección y perder la capacidad de correlación entre el resultado medido y la realidad del fenómeno en estudio.

Podemos concluir que existen diferentes escalas para medir la calidad de vida en las personas, las más utilizadas son la SF-36 y KDQOL 36, sin embargo los pacientes con ERC tienen características y condiciones especiales por lo cual se recomienda emplear herramientas específicas para evaluar su condición real. La escala KDQOL 36, posee dimensiones específicas para evaluar al paciente con enfermedad renal, sus características psicométricas en cuanto a reproducibilidad, validez y sensibilidad son confiables, y su utilidad es adecuada ya que es de fácil aplicación, poca complejidad y bajo costo, esta escala se encuentra adaptada transculturalmente en varios países de habla hispana, por lo cual la se hace recomendable para evaluar la calidad de vida en población con enfermedad renal.

Recibido: 5 mayo 2018

Revisado: 11 junio 2018

Modificado: 27 agosto 2018

Aceptado: 5 septiembre 2018

\section{Bibliografía}

1. Organización Panamericana de la Salud/ Organización Mundial de la Salud [Internet]. Washington DC: [Consultado 10 enero 2018]. Disponible en: http:// www.paho.org/hq/index.php?option=com_content\&view=article\&id=10542\%3A2015-opsoms-sociedad-latinoamericana-nefrologia-enfermedad-renal-mejorar-tratamiento\&Itemid=1926\&la ng=es.

2. Ázar Arroyo L, Orte Martínez R, Otero González A. Enfermedad Renal Crónica Avanzada. Nefrología. 2008; 28 (Supl 3):S3-6.

3. Cuevas Budhart MÁ, Saucedo García RP, Romero Quechol G, García Larumbe JA, Hernández $P$, Puente A. Relación entre las complicaciones y la calidad de vida del paciente en hemodiálisis. Enferm Nefrol [Internet]. 2017 [Consultado 20 enero 2018];20(2):112-119. Disponible en: http:// scielo.isciii.es/pdf/enefro/v20n2/2255-3517-enefro-20-02-00112.pdf

4. Barros Higgins L, Herazo Beltrán $Y$, Aroca Martínez G. Calidad de vida relacionada con la salud en pacientes con enfermedad renal crónica. Rev. Fac. Med [Internet]. 2015 [Consultado 25 enero 2018];63(4):641-641. Disponible en: https:// revistas.unal.edu.co/index.php/revfacmed/article/ view/49805/54037

5. García Llana H, Remor E, del Pero G, Selgas R. El papel de la depresión, la ansiedad, el estrés y la adhesión al tratamiento en la calidad de vida relacionada con la salud en pacientes en diálisis: revisión sistemática de la literatura. Revista Nefrología [Internet]. 2014 [Consultado 15 febrero 2018]; 34(5):637-57. Disponible en: http://scielo.isciii.es/ pdf/nefrologia/v34n5/revisionl.pdf

6. Moher D, Liberati A, Tetzlaff J, Altman GD, The prisma Group. Preferred Reporting Items for Systematic Reviews and Meta-Analyses: The PRISMA Statement. Plos medicine [Internet]. 2009 [Consultado 1 febrero 2018];6(7):1-6. Disponible en: https:// www.ncbi.nlm.nih.gov/pmc/articles/PMC2707599/ pdf/pmed.1000097.pdf

7. Vasconcelos CM, da C.B, Backes V.S.M, Gue JM. La evaluación en la enseñanza de grado en enfermería en América Latina: una revisión integrativa. 
Enfermería Global [Internet]. 2011 [Consultado 14 marzo 2018];10(28):96-117. Disponible en: http:// scielo.isciii.es/pdf/eg/v10n23/docencia2.pdf

8. Guirao Goris A, Cabrero García J, Moreno Pina JP, Muñoz Mendoza CL. Revisión estructurada de los cuestionarios y escalas que miden la actividad física en los adultos mayores y ancianos. Gac Sanit [Internet]. 2009 [Consultado 20 marzo 2018];23(4):334-67. Disponible en: http:// scielo.isciii.es/scielo.php?script=sci_arttext\&pi$d=$ S1695-61412011000300008

9. Campo Arias A, Oviedo H. Propiedades Psicométricas de una Escala: la Consistencia Interna. Rev Salud Pública [Internet]. 2008 [Consultado 25 febrero 2018];10(5):831-9. Disponible en: http://www. redalyc. org/articulo. $0 a ? \mathrm{id}=42210515$

10. Sánchez R, Echeverry J.Validación de escalas de medición en salud. Rev Salud Publica [Internet]. 2004 [Consultado 14 marzo 2018];6(3):302-18. Disponible en: https://www.scielosp.org/pdf/rsap/2004. v6n3/302-318/es

11. Guillemin F, Bombardier C, Beaton DC. Cultural Adaptation of Life Measures: Literature Review and Proposed Guidelines. Clin Epidemiol [Internet]. 1993 [Consultado 19 septiembre 2018];46(12): 1417-32. Disponible en: https://pdfs.semanticscholar.org/a2a8/fc3722ce868ac3cc37fd539f50afa3lf4445.pdf

12. Amro A, Waldum B, Dammen T, Miaskowski C, Os L. Symptom clusters in patients on dialysis and their association with quality-of-life outcomes. Journal of Renal Care [Internet]. 2014 [Consultado 18 septiembre 2018];40(1):23-33. Disponible en: http://catalogo.fucsalud.edu.co:2164/ehost/pdfviewer/pdfviewer?vid=18\&sid=940dfc58-4e94-4d df-8128-0180bb7cla9f\%40pdc-v-sessmgr01

13. Anees M, Ibrahim M, Imtiaz M, Batool S, Elahi I, Malik M. Translation, Validation and Reliability of the Kidney Diseases Quality of Life-Short Form (KDQOL-SF Form) Tool in Urdu. Journal of the College of Physicians and Surgeons Pakistan [Internet]. 2016 [Consultado 20 marzo 2018]; 26(8): 651-4. Disponible en: http://catalogo.fucsalud.edu. co:2164/ehost/pdfviewer/pdfviewer?vid=11\&sid=940dfc58-4e94-4ddf-8128-0180bb7cla9f\%40pdc-v-sessmgr01
14. Ayoub A, Nelson K, Wood P. Cultural relevance of the quality-of-life tools for people with kidney failure. J Ren Care [internet]. 2013 [Consultado 14 marzo 2018];39(4):236-45. Disponible en: http://catalogo.fucsalud.edu.co:2164/ehost/pdfviewer/pdfviewer?vid=6\&sid=940dfc58-4e94-4ddf -8128-0180bb7cla9f\%40pdc-v-sessmgr01

15. Barbero Narvona E, Tejeda Araez E, Herrera Morales C, Montserrat García S, Gascó Coscojuela N, Junyent Iglesias E. Estudio comparativo del estado físico, mental y percepción de calidad de vida relacionada con la salud de los pacientes en diálisis. Enferm Nefrol [Internet]. 2016 [Consultado 16 marzo 2018];19(1):29-35. Disponible en: http://catalogo. fucsalud.edu.co:2164/ehost/pdfviewer/pdfviewer?vid $=2 \&$ sid $=940 \mathrm{dfc} 58-4 \mathrm{e} 94-4 \mathrm{ddf}-8128-0180 \mathrm{bb}$ 7cla9f\%40pdc-v-sessmgr01

16. Chao $S$, Yen $M$, Lin $T$, Sung J, Wang $M$, Hung $S$. Psychometric Properties of the Kidney Disease Quality of Life-36 Questionnaire (KDQOL-36 ${ }^{\mathrm{TM}}$ ). Western Journal of Nursing Research [Internet]. 2016 [Consultado 14 marzo 2018];38(8):1067-82. Disponible en: https://www.ncbi.nlm.nih.gov/pmc/ articles/PMC4274701/

17. Chen JY, Choi EP, Wan EY, Chan AK, Tsang JP. Chan $\mathrm{KH}$, et al. Validation of the Disease-Specific Components of the Kidney Disease Quality of Life36 (KDQOL-36) in Chinese Patients Undergoing Maintenance Dialysis. Plos One [Internet]. 2016 [Consultado 20 marzo 2018]; 11(5): 1-13. Disponible en: http://catalogo.fucsalud.edu.co:2164/ ehost $/$ pdfviewer $/$ pdfviewer? vid=37\&sid=940dfc58-4e94-4ddf-8128-0180bb7cla9f\%40pdc-v-sessmgr01

18. Chow SKY, Tam BML. Is the kidney disease quality of life-36 (KDQOL-36) a valid instrument for Chinese dialysis patients?. BMC Nephrology [Internet]. 2014 [Consultado 23 enero 2018];15(199): 1-7. Disponible en: https://bmcnephrol.biomedcentral.com/track/pdf/10.1186/1471-2369-15-199

19. Pina Pereira $R$, Aparecido Batista $M$, de Sousa Meira A, Pilotto de Oliveira M, Kusumota L. Calidad de vida de ancianos con enfermedad renal crónica en tratamiento conservador. Revista Brasileira de Enfermagem [Internet]. 2017 [Consultado 13 marzo 2018]; 70(4):851-9. Disponible en: http:// www.scielo.br/scielo.php?script=sci_arttext\&pi- 
$d=S 0034-71672017000400851 \& \operatorname{lng}=e n \& n r \quad m=i-$ so\&tlng=en

20. Dehesa López E, Correa Rotter R, Olvera CastiIlo D, González Parra C, Baizabal Olarte R, Orozco Vega R. Transcultural adaptation and validation of the Mexican version of the kidney disease questionnaire KDQOL-SF36 versión 1.3. Qual Life Res [Internet]. 2017 [Consultado 21 febrero 2018];26(1):193-8. Disponible en: http:// catalogo.fucsalud.edu.co:2161/ehost/pdfviewer/ pdfviewer? vid=15\&sid=8a922b2b-1093-4d 9f-8e3e-ce7649465691\%40sessionmgr4008

21. Erez G, Selman L, Murtagh FEM. Measuring health-related quality of life in patients with conservatively managed stage 5 chronic kidney disease: limitations of the Medical Outcomes Study Short Form 36: SF-36. Qual Life Res [Internet]. 2016 [Consultado 24 marzo 2017]; 25(1):2799-809. Disponible en: http://catalogo.fucsalud.edu.co:2161/ehost/pdfviewer/pdfviewer?vid=19\&sid=8a922b2b-1093-4d 9f-8e3e-ce7649465691\%40sessionmgr4008

22. Twomey JC, PhD, RN, Barrett BJ, MB, MSc, Et al. Psychometric Properties of the Patient's Perception of Life on Hemodialysis Scale. Journal of Nursing Measurement [Internet]. 2015 [Consultado 15 febrero 2018]; 21(1):72-81. Disponible en: https://catalogo.fucsalud.edu.co:2057/ docview/1673956920/fulltextPDF/BC58CFAEBB7B4E6FPQ/ 1?accountid=107581

23. Lopez JM, Fukushima RLM, Inouye $K$, Iost Pavarini SC, Souza Orlandi F. Quality of life related to the health of chronic renal failure patients on dialysis. Acta Paul Enferm [Internet]. 2014 [Consultado 16 marzo 2018];27(3):230-6. Disponible en: http://www.scielo.br/scielo.php?pi$d=$ S0103-21002014000300230\&script=sci_arttext\&tlng=en

24. Mateti UV, Nagappa AN, Attur RP, Nagaraju SP, Mayya SS, Balkrishnan R. Cross-Cultural Adaptation, Validation and Reliability of the South Indian (Kannada) Version of the Kidney Disease and Quality of Life (KDQOL-36) Instrument. Saudi J Kidney Dis Transpl [Internet]. 2015 [Consultado 20 marzo 2018];26(6):1246-52. Disponible en: http://catalogo.fucsalud.edu.co:2161/ehost/pdfviewer/pdfviewer?vid=38\&sid=8a922b2b-1093-4d 9f-8e3e-ce7649465691\%40sessionmgr4008
25. Hacker E, Lora CM, Ackerson L, De Salvo KB, Et al. Valídatín of the kidney disease quality oe life

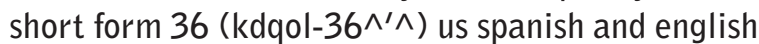
versions in a cohort of hispanics with chronic kidney disease. Ethnicity \& Disease [Internet]. 2013 [Consultado 17 abril 2018];23(2):202-9. Disponible en: http://catalogo.fucsalud.edu.co:2161/ ehost/pdfviewer/pdfviewer?vid=43\&sid=8a922b2b-1093-4d 9f-8e3e-ce7649465691\%40sessionmgr 4008

26. Ruiz Parrado MdC, Gómez Pozo M, Crespo Garrido M, Tierno Tendero C, Crespo Montero R. Análisis de la calidad de vida del paciente en la etapa prediálisis. Enfermería Nefrológica [Internet].2017 [Consultado 3 febrero 2018];20(3):233-40. Disponible en: http:// www.redalyc.org/articulo.oa?id=359852875006

27. Tao $X$, Chow $S$, Wong F. Determining the validity and reliability of the Chinese version of the Kidney $\mathrm{Di}$ sease Quality of Life Questionnaire (KDQOL-36 ${ }^{\mathrm{TM}}$ ). BMC Nephrology [Internet]. 2014 [Consultado 20 Marzo 2018]; 15(115):1-9. Disponible en: http://catalogo.fucsalud.edu.co:2161/ehost/pdfviewer/pdfviewer?vid=51\&sid=8a922b2b-1093-4d 9f-8e3e-ce7649465691\%40sessionmgr4008

28. Loosman $W L$, Hoekstra $T$, van Dijk $S$, Terwee $C B$, Honig A, Siegert CE, Dekker FW. Short-Form 12 or Short-Form 36 to measure quality-of-life changes in dialysis patients?. Nephrol Dial Transplant. [Internet]. 2015 [Consultado 27 febrero 2018]; 30(7): 1170-6. Disponible en: https://www.ncbi.nlm.nih. gov/pubmed/25829325

29. Chaves k, Duarte A, Vesga J. Adaptación transcultural del cuestionario KDQOL SF 36 para evaluar calidad de vida en pacientes con enfermedad renal crónica en Colombia. Rev.Fac. Med [Internet]. 2013 [Consultado 22 marzo 2018];21(2):34-42. Disponible en: http://www.scielo.org.co/pdf/med/v2ln2/ v2ln2a04.pdf

30. Urzúa M A, Navarrete M. Calidad de vida en adultos mayores: análisis factoriales de las versiones abreviadas del WHOQoL-Old en población chilena. Rev. méd. Chile [Internet]. 2013 [Consultado 25 Abril 2018];141(1):28-33. Disponible en: https:// scielo.conicyt.cl/scielo.php?script=sci_arttext\&pi$d=$ S0034-98872013000100004\&Ing=es. 
31. Vera Villarroel P, Silva J, Celis Atenas K, Pavez P. Evaluación del cuestionario SF-12: verificación de la utilidad de la escala salud mental. Rev. méd. Chile [Internet]. 2014 [Consultado 25 abril 2018];142(10):1275-83. Disponible en: https:// scielo.conicyt.cl/scielo.php?script=sci_arttext\&pi$d=$ S0034-98872014001000007\&lng=es.

32. Vilagut $G$, Ferrer $M$, Rajmil L, Rebollo $P$, Permanyer Miralda G, Quintana JM, et al. El Cuestionario de Salud SF-36 español: una década de experiencia y nuevos desarrollos. Gac Sanit [Internet]. 2005 [Consultado 26 abril 2018]; 19(2):135-50. Disponible en: http://scielo.isciii.es/scielo.php?script=sci_arttext\&pid=S0213-91112005000200007\&In$g=e s$.

33. Espinoza I, Osorio P, Torrejón M, Lucas R, Bunout D. Validación del cuestionario de calidad de vida (WHOQOL-BREF) en adultos mayores chilenos. Rev. méd. Chile [Internet]. 2011 Mayo [Consultado 03 febrero 2018];139(5):579-86. Disponible en: https:// scielo.conicyt.cl/scielo.php?script=sci_arttext\&pi$d=$ S0034-98872011000500003\&lng=es.
34. Cardona Arias JA, Higuita Gutiérrez LF. Aplicaciones de un instrumento diseñado por la OMS para la evaluación de la calidad de vida. Rev Cubana Salud Pública [Internet]. 2014 [Consultado 26 abril 2018]; 40(2):175-89. Disponible en: http:// scielo.sld.cu/scielo.php?script=sci_arttext\&pi$d=$ S0864-34662014000200003\&lng=es.

35. Lujan T, Cardona A. Construcción y validación de escalas de medición en salud: revisión de propiedades psicométricas. Archivos de medicina, [Internet]. 2015 [Consultado 26 abril 2018];11(3). Disponible en: http://www.archivosdemedicina.com/ medicina-de-familia/construccin-y-validacin-deescalasde-medicin-en-salud-revisin-depropiedadespsicomtricas.php?aid=6694.

Este artículo se distribuye bajo una Licencia Creative Commons Atribución-NoComercial 4.0 Internacional. https://creativecommons.org/licenses/by-nc/4.0/ 